\title{
Cinema brasileiro e o ensino dos transtornos da personalidade
}

\author{
Brazilian cinema and the teaching of personality disorders
}

\author{
Tabata Galindo Honorato ${ }^{1,2}$ (1) galindo.tabata@gmail.com \\ Maria Cristina Mazzaia' (1) mazzaia@terra.com.br \\ Amanda Carolina Franciscatto Avezani1 (D) amandaavezani@gmail.com \\ Francisco Lotufo Neto ${ }^{2}$ (D) franciscolotufo@gmail.com
}

\begin{abstract}
RESUMO
Introdução: O uso de filmes comerciais em sala de aula é uma prática comum e acessível. A prática de exibição de filmes para o ensino é definida pelo termo cinemeducation.

Objetivo: O presente estudo partiu da hipótese de que o cinemeducation, como metodologia ativa, poderia contribuir para o aprendizado dos transtornos da personalidade (TP) na graduação. Foram ministradas aulas presenciais para 213 estudantes de Medicina, Enfermagem, Psicologia,
\end{abstract} Fisioterapia e Fonoaudiologia, para avaliar o conhecimento desenvolvido sobre TP.

Método: O estudo é transversal e quantitativo, composto por amostras pareadas e dependentes (antes e depois). As etapas foram: 1. aplicação de instrumento avaliativo em forma de questionário (antes); 2. aula composta por exibição de cenas de filmes brasileiros e reflexão e discussão sobre elas; 3. aplicação do mesmo questionário (depois); e 4. análise estatística comparativa entre os resultados.

Resultados: O método se mostrou efetivo para o processo ensino-aprendizagem, havendo melhora autorreferida no conhecimento dos estudantes após a aula (questão 1) e melhora observável ao identificarem e conceituarem os TP (questão 3). Além disso, os estudantes referiram, em média, que a estratégia contribuía para a aprendizagem, antes da aula, e mantiveram em média essa opinião, depois (questão 2).

Conclusões: Alcançou-se o objetivo proposto porque se utilizou amostra estatisticamente significativa de estudantes, e os resultados confirmaram que o método é efetivo para o ensino. Além disso, a discussão evidenciou que o uso dos filmes pode contribuir para o o aprendizado, condizendo com as características de estudantes da geração atual, uma vez que valoriza o uso de tecnologias em sala de aula e permite o aprendizado crítico-reflexivo e a participação ativa dos sujeitos. As limitações do estudo se referem à escassez de filmes brasileiros contemporâneos que exemplifiquem todos os TP, não havendo cenas representativas para TP esquizotípica, dependente e esquiva. Além disso, considera-se necessário estudo comparativo entre o método tradicional de ensino dos TP e o cinemeducation, de forma a investigar a eficácia deste quando comparado às aulas tradicionais.

Palavras-chave: Transtornos da Personalidade; Filmes Cinematográficos; Filmes Educativos; Ensino; Aprendizagem.

\section{ABSTRACT}

Introduction: The use of commercial films in the classroom is a common and accessible practice. The practice of showing films for teaching is defined by the term cinemeducation.

Objective: The present study was based on the hypothesis that cinemeducation, as an active methodology, could contribute to the learning of Personality Disorders $(P D)$ during undergraduate training. Face-to-face classes were given to 213 students of medicine, nursing, psychology, physiotherapy and speech therapy, to assess the knowledge developed about PD.

Method: The study is cross-sectional and quantitative, composed of paired and dependent samples (before and after). The steps were: a) application of an assessment tool in the form of a questionnaire (before); b) class, consisting of exhibition, reflection and discussion of scenes from Brazilian films, c) application of the same questionnaire (after), and d) comparative statistical analysis between the results.

Results: The results showed that the method is effective for the teaching-learning process, with self-reported improvement in students' knowledge after class (question 1), and an observable improvement when identifying and conceptualizing PD (question 3). Furthermore, students reported, on average, that the strategy contributed to learning, before class, and they maintained that opinion, on average, afterwards (question 2).

Conclusions: The proposed objective was achieved by using a statistically significant sample of students and the results confirmed that the method is effective for teaching. The discussion showed that the use of films can improve learning, and it is consistent with the characteristics of students of the actual generation, since it values the use of technologies in the classroom, allowing critical-reflective teaching and the active student participation. The limitations of the study include the scarcity of contemporary Brazilian films that exemplify all PDs, with no representative scenes for schizotypical, dependent and avoidant PD. Furthermore, a comparative study between the traditional method of teaching PD and cinemeducation is considered necessary in order to investigate its effectiveness when compared to traditional classes.

Keywords: Personality Disorders; Motion Pictures; Teaching Materials; Teaching; Learning.

1 Universidade Federal de São Paulo, São Paulo, São Paulo, Brasil.

2 Universidade de São Paulo, São Paulo, São Paulo, Brasil.

Editora-chefe: Daniela Chiesa

| Editora associada: Rosiane Viana Zuza Diniz

Recebido em 13/07/20; Aceito em 14/04/21. | Avaliado pelo processo de double blind review. 


\section{INTRODUÇÃO}

O uso de filmes comerciais em sala de aula é uma prática comum e acessível. Assistir a um filme com objetivo didático-pedagógico costuma ser uma atividade prazerosa, uma vez que associa o lazer ao aprendizado'. A prática de exibição de filmes para o ensino em cursos da área da saúde é definida pelo termo cinemeducation ${ }^{2}$.

Os primeiros estudos que abordaram o tema cinema e educação em saúde datam da década de 1980. Atualmente, as publicações sobre cinemeducation provêm de variadas especialidades clínicas, a exemplo da geriatria, saúde pública e saúde mental, e psiquiatria, sendo esta última o tema mais frequente ${ }^{3}$.

A proposta de utilização defilmes para o desenvolvimento de conteúdos referentes à área de saúde mental atende à demanda de formação de profissionais de saúde críticos e reflexivos, e, para isso, são necessários processos de ensinoaprendizagem que promovam a construção do conhecimento pelo próprio estudante. Os métodos ativos de ensino são alternativas fundamentais que respondem a essa necessidade, uma vez que esses recursos favorecem a socialização do conhecimento e o desenvolvimento de autonomia, e suscitam a curiosidade do estudante, desenvolvendo, por fim, capacidades e competências para tomada de decisão, tanto individuais como em equipe, ao aproximarem o estudante da prática social, ou seja, de seus contextos de vida 4 .

As metodologias ativas, no que se refere à educação profissional, inserem o estudante como protagonista do processo ensino-aprendizagem, o que exige mudanças de postura e desenvolvimento de responsabilidade sobre o seu processo de formação ${ }^{5}$.

Os estudos voltados para um método facilitador no ensino da saúde mental podem convergir com as dificuldades inerentes aos conteúdos complexos e subjetivos, tais como o diagnóstico psiquiátrico ou a própria psicopatologia. Algumas iniciativas já se apropriaram de filmes de ficção com o propósito de torná-los um recurso para o ensino da psicopatologia6. A viabilidade desse método só foi possível em virtude do constante interesse que o cinema mostrou em relação aos diversos transtornos mentais ${ }^{7,8}$.

Dentre os transtornos mentais, o estudo dos transtornos da personalidade (TP) apresenta muitas particularidades. A personalidade é uma característica humana de difícil conceituação, e sua definição se modifica ao longo do tempo, de acordo com o contexto sociocultural. Atualmente, é possível definir a personalidade como um traço duradouro, um estado condicionado ao momento existencial do indivíduo, influenciado pelos componentes genéticos e pelas interações sociais ${ }^{9}$.

Os TP se caracterizam, portanto, como um padrão persistente e inflexível de experiência interna e comportamental divergente da cultura do indivíduo, que pode levar a sofrimento e prejuízos na vida. Os aspectos comprometidos podem ser cognitivos, afetivos, interpessoais e da impulsividade ${ }^{10}$.

Dado que a personalidade é infinitamente variável, não é possível determinar um enquadramento padrão para um indivíduo existir, sentir e se comportar. Assim sendo, quando se introduz o conteúdo sobre TP nos cursos da área da saúde, é possível que os métodos tradicionais de ensino não o abarquem em toda sua amplitude. O presente estudo partiu da hipótese de que métodos ativos, que se associem ao universo artístico e tecnológico, tal qual o cinemeducation, poderiam contribuir para o aprendizado dos TP.

Assim, questiona-se:

- $\quad$ Existe aprendizado sobre o tema TP após utilização de cenas de filmes, seguida por reflexão e discussão sobre as cenas apresentadas?

Para responder a essa questão, pretendeu-se avaliar o conhecimento de estudantes de cursos de graduação em saúde acerca dos TP, o que passa a ser descrito a seguir.

Portanto, o presente estudo tem como objetivo avaliar o conhecimento de estudantes de graduação, em momentos antes e depois de uma aula, sobre a caracterização dos TP, a partir da utilização de cenas de filmes brasileiros como estratégia de ensino.

\section{MÉTODOS}

Trata-se de estudo transversal, de natureza quantitativa, composto por amostras pareadas e dependentes. As etapas do processo se caracterizaram por: 1. aplicação de instrumento avaliativo em forma de questionário aos estudantes (antes); 2. apresentação de aula composta por cenas de filmes brasileiros e reflexão e discussão sobre o tema; 3. aplicação do mesmo questionário aos estudantes (depois); e 4. análise estatística comparativa entre os resultados.

As aulas foram realizadas pelos dois pesquisadores principais, durante os meses de agosto, setembro e outubro de 2019, para turmas de universidades públicas - Universidade de São Paulo (USP) e Universidade Federal de São Paulo (Unifesp) - de diferentes cursos da área da saúde. As aulas citadas apresentaram o mesmo conteúdo e as mesmas estratégias de ensino em todos os cursos. No início das aulas, os estudantes foram esclarecidos sobre o método de avaliação dos conteúdos veiculados em aula, por meio do questionário aplicado antes e depois da aula.

A amostra mínima para o estudo foi obtida por meio de cálculo amostral, correspondente à fórmula para amostras pareadas (antes edepois), a seguir: $n=\frac{\left(2 \cdot \sigma_{\mathrm{d}}^{2}\right) \cdot\left(\mathrm{z}_{\alpha / 2}+\mathrm{z}_{1-\beta}\right)^{2}}{\Delta^{2}} \cdot \mathrm{A}$ fórmula foi aplicada com base nos resultados das três perguntas 
do questionário. A partir dos dados de 213 indivíduos, testouse a hipótese de, em média, a diferença entre a pontuação do período depois e do período antes ser de 4 pontos, com nível de significância de $5 \%$ e poder do teste de $80 \%$. Após os cálculos, obteve-se o seguinte resultado: para a questão 1, foi verificado que $\mathrm{s}_{\mathrm{d}}^{2}=4,842723$ e $n \approx 5$; para questão $2, \mathrm{~s}_{\mathrm{d}}^{2}=6,173974666$ e $n \approx 7$; para questão $3, \mathrm{~s}_{\mathrm{d}}^{2}=8,472673$ e $n \approx 9$. Considerando o maior $\mathrm{n}$ para o tamanho amostral, tem-se que 9 é o tamanho suficiente de indivíduos, sendo 9 medidas antes e 9 depois. No total, houve 213 medidas antes e 213 depois, correspondentes aos questionários respondidos por 213 estudantes de graduação de cursos de: Medicina (20), Psicologia (74), Enfermagem (52), Fonoaudiologia (35) e Fisioterapia (32). Os estudantes foram orientados sobre a possibilidade de utilização dos resultados dos questionários para publicação científica, referente ao tema da pesquisa, bem como sobre o sigilo de suas identidades.

Sobre os critérios de inclusão, os participantes foram os estudantes que estavam presentes no momento da aula, que concordaram tanto em participar quanto com a utilização dos resultados do questionário como dados de pesquisa. Eles receberam e leram o Termo de Consentimento Livre e Esclarecido (TCLE) e ficaram em posse de uma via assinada pelo pesquisador responsável. A pesquisa seguiu a Resolução no 466/2012 do Conselho Nacional de Saúde do Ministério da Saúde (CNS/MS), que trata da pesquisa envolvendo seres humanos, e foi aprovada pelo Comitê de Ética da Unifesp: Certificado de Apresentação para Apreciação Ética (CAAE) no 11935519.3.0000.5505.

A aula ministrada aos estudantes se baseou na exibição de cenas de filmes brasileiros contemporâneos, e, a seguir, houve espaço para reflexão e discussão, com o intuito de problematizar as características dos TP, quando associadas aos comportamentos e ao contexto sócio-histórico-cultural apresentado pelos personagens participantes das cenas. As cenas dos filmes foram previamente editadas e validadas em estudo anterior, que avaliou diversos personagens do cinema brasileiro, identificando aqueles que apresentavam padrões comportamentais/diagnóstico de TP11. No Quadro 1, podemse visualizar os títulos dos filmes utilizados e seus respectivos personagens e intérpretes protagonistas em cada cena.

$\mathrm{O}$ instrumento que possibilitou avaliação do conhecimento antes e depois da aula foi criado pelos pesquisadores em forma de questionário:

- Questão 1, em uma escala Likert de 10 pontos: "Qual é o seu nível de conhecimento sobre os transtornos da personalidade?".

- Questão 2, também em escala Likert de 10 pontos: "Em termos quantitativos, como o uso de obras artísticas pode contribuir para o aprendizado de transtornos da personalidade?"

- $\quad$ Questão 3, uma associação entre conceitos: “Associe o transtorno da personalidade à sua definição".

O software utilizado para a análise dos dados, por meio de cálculos e testes estatísticos, foi o $\mathrm{R}$ versão $4.0 .1^{\circledR}$. Considerando a grande amostra, optou-se pela comparação de médias entre os grupos, por meio dos testes: $t$ de Student, quando os dados seguem a normalidade, Mann-Whitney, quando são independentes, e Wilcoxon dos postos assinalados, quando são dependentes, de forma a averiguar diferença ou não entre os grupos.

No que concerne à linha teórica, a pesquisa é norteada pelo conteúdo do Manual diagnóstico e estatístico de transtornos mentais (Diagnostic and statistical manual of mental disorders - DSM-5), por meio do qual foram delineadas a definição diagnóstica dos personagens e a escolha das cenas para composição da aula, bem como a construção do instrumento de avaliação $^{10}$.

Quadro 1. Identificação dos filmes utilizados, de acordo com personagem, ator ou atriz e transtorno da personalidade - São Paulo ${ }^{11}$.

\begin{tabular}{lccc}
\hline \multicolumn{1}{c}{ FILME } & PERSONAGEM & ATOR/ATRIZ & $\begin{array}{c}\text { TRANSTORNO DA } \\
\text { PERSONALIDADE }\end{array}$ \\
\hline É proibido fumar (2009) & Baby & Glória Pires & Paranoide \\
Á beira do caminho (2012) & João & João Miguel & Esquizoide \\
O invasor (2001) & Anízio & Paulo Miklos & Antissocial \\
Nome próprio (2007) & Camila & Leandra Leal & Borderline \\
O auto da compadecida (2000) & Dora & Denise Fraga & Histriônica \\
2 perdidos numa noite suja (2002) & Paco & Débora Falabella & Narcisista \\
Tropa de elite (2007) & Nascimento & Wagner Moura & Obsessivo-Compulsivo \\
\hline
\end{tabular}

Fonte: Honorato, 2018. 


\section{RESULTADOS}

Foram avaliados os dados obtidos nas três questões, de forma comparativa, como amostras pareadas (antes e depois), sendo a intervenção configurada em aula baseada em cenas de filmes como estratégia deensino (cinemeducation). Usaram-setestes paramétricos e não paramétricos para comparar as pontuações (antes e depois). Como a intenção era justamente comparar o nível de conhecimento entre o antes e o depois, quando havia a significância dos testes, observou-se se a média dos grupos de notas apresentava nota média maior. Os resultados foram expostos de forma descritiva e inferencial, e são apresentados a seguir.

No Gráfico 1, de autoria dos próprios pesquisadores, referente à questão 1 - "Qual é o seu nível de conhecimento sobre transtornos da personalidade?" -, percebe-se que as pontuações depois aparentam ser maiores que as de antes e parecem se comportar de forma diferente. A pontuação antes vai de 0 a 8 com mediana em 3, já a pontuação depois vai de 3 a 10 com pontuação mediana de 7. Quando se realizou um teste não paramétrico (teste dos postos assinalados de Wilcoxon), verificouse que as pontuações se comportam de maneira diferente, antes e depois, ( $p$-valor < 2,2 × 10-16), sendo a pontuação média antes de 3,239437 e depois de 6,849765. Assim, depois da intervenção, há uma melhora (3,61 pontos) no desempenho médio das pessoas e também no desempenho mediano (4 pontos). Considerando intervalo de confiança (IC) de 95\%, com relação à média da diferença das notas antes e depois, obtiveram-se -3,819 e -3,401 para a nota média de depois ser maior que antes.

De acordo com o Gráfico 2, de autoria dos próprios pesquisadores, referente à questão 2 ("Em termos quantitativos, como o uso de obras artísticas pode contribuir para o aprendizado de transtornos da personalidade?"), há, nos períodos antes e depois, pelo menos dois indivíduos com pontuações consideravelmente inferiores aos demais (outliers). Também se observa que não há diferença aparente entre as notas dos grupos. A pontuação antes vai de 0 a 10 com mediana em 8 , já a pontuação depois vai de 1 a 10 com mediana em

Gráfico 1. Boxplot com resultados da questão 1 ("Qual é o seu nível de conhecimento sobre transtornos da personalidade?") antes e depois.

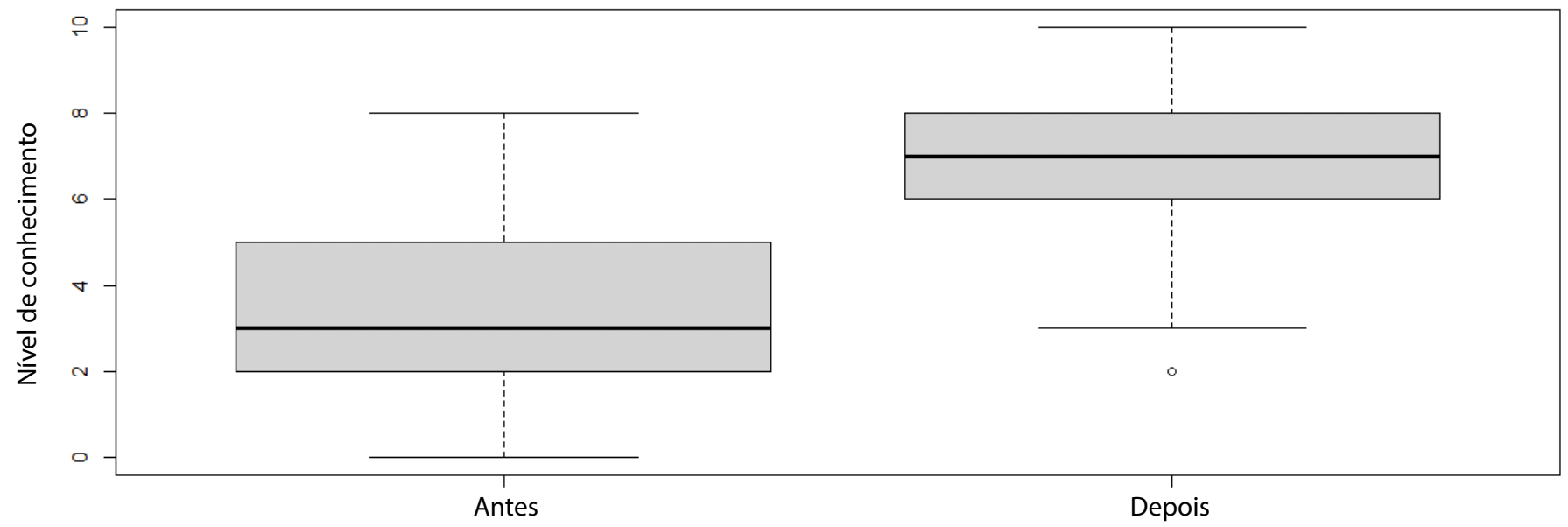

Gráfico 2. Boxplot com resultados da questão 2 ("Em termos quantitativos, como o uso de obras artísticas pode contribuir para o aprendizado de transtornos da personalidade?") antes e depois.

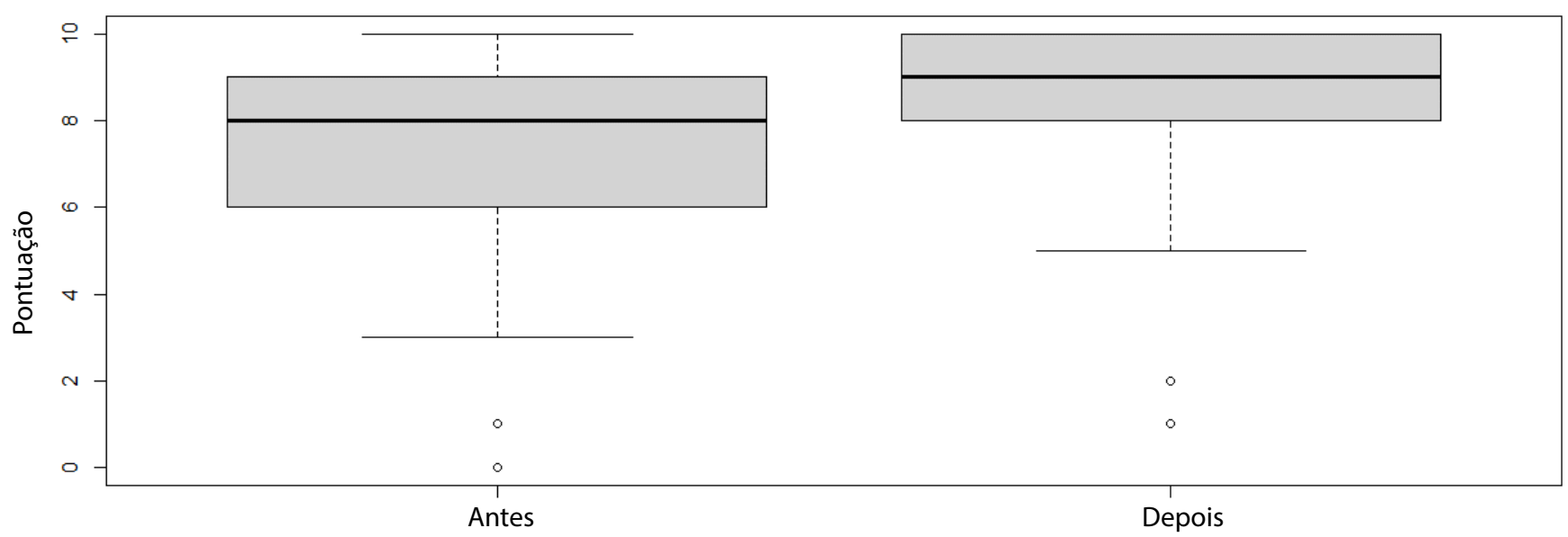


9. Mas, quando se realizou o teste dos postos assinalados de Wilcoxon, as pontuações se comportaram de forma diferente $(p$-valor $=1,037 \times 10-13)$, sendo a pontuação média antes de 7,366197 e depois de 8,723005 . Com um IC de $95 \%$, a nota média antes está entre -1,593 e -1,120, ou seja, menor do que depois. Assim, houve aumento das notas depois.

Na Gráfico 3, de autoria dos próprios pesquisadores, referente à questão 3 ("Associe o transtorno da personalidade à sua definição"), a pontuação do grupo antes vai de 0 a 10 com mediana em 5, já a pontuação depois vai de 3 a 10 com mediana em 8. As pontuações depois parecem ser maiores que as pontuações antes. Pelo teste dos postos assinalados de Wilcoxon, as pontuações se comportam de forma diferente antes e depois ( $p$-valor $<2,2 \times 10-16$ ), sendo a média antes de 4,985915 com mediana 5; e depois de 8,046948 com mediana 8. Assim, há uma melhora média (3,06 pontos) e na mediana (3 pontos) quanto ao desempenho das pessoas após a intervenção. Com um IC de 95\%, a nota média antes está entre $-3,338$ e -2,784, menor que depois. Assim, houve melhora nas notas no momento depois.

No Gráfico 4, de autoria dos próprios pesquisadores, observa-se que a quantidade de acertos aparenta ser consideravelmente maior que a quantidade de erros, para a questão 3, no momento depois da aula. No total, de 213 medidas depois, para questão 3, houve 1.725 acertos e 405 erros. Quando se realizou o teste t de Student, tendo como base esses dados, verificou-se que há diferença nas médias de acertos e erros no momento depois ( $p$-valor $=3,718 \times 10-8$ ), sendo maior a quantidade média de acertos de 172,5 , quando comparada à quantidade média de erros de 40,5. Como o zero não está no intervalo e o intervalo é positivo, tem-se que a quantidade de acertos é maior que a quantidade de erros, sendo a média da diferença de acertos e erros entre 101,535 e 162,465 a 5\% de significância.

Gráfico 3. Boxplot com resultados da questão 3 ("Associe o transtorno da personalidade à sua definição") antes e depois.

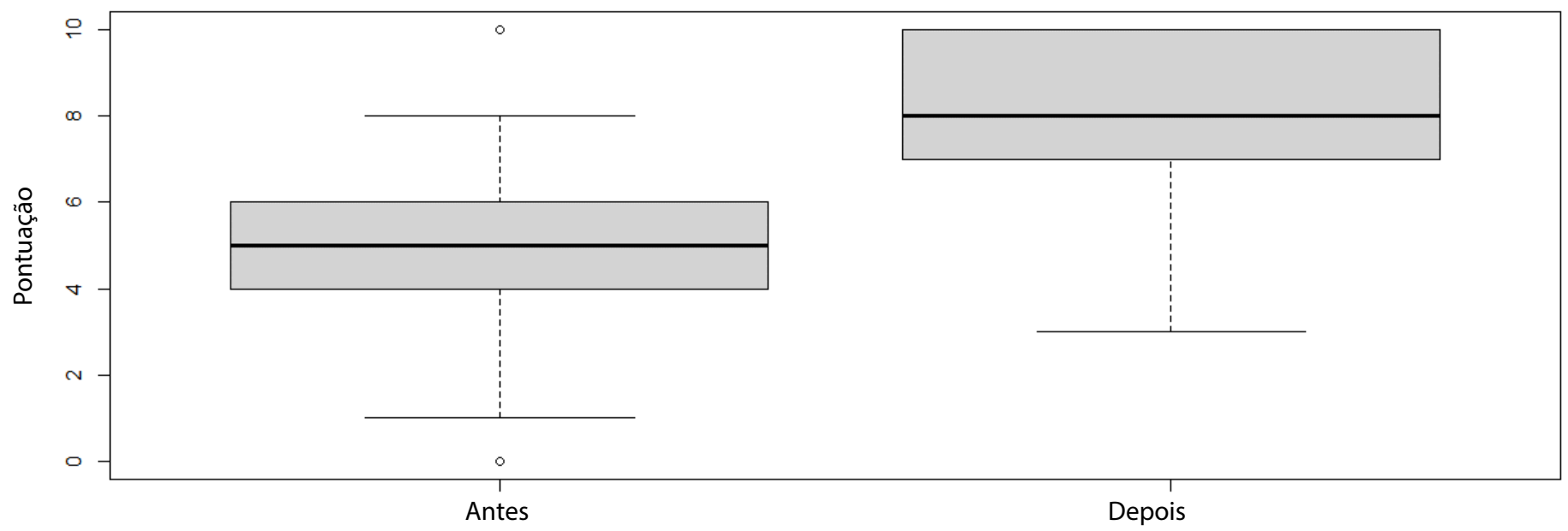

Gráfico 4. Boxplot com quantidade de acertos e erros totais para a questão 3 ("Associe o transtorno da personalidade à sua definição") depois.

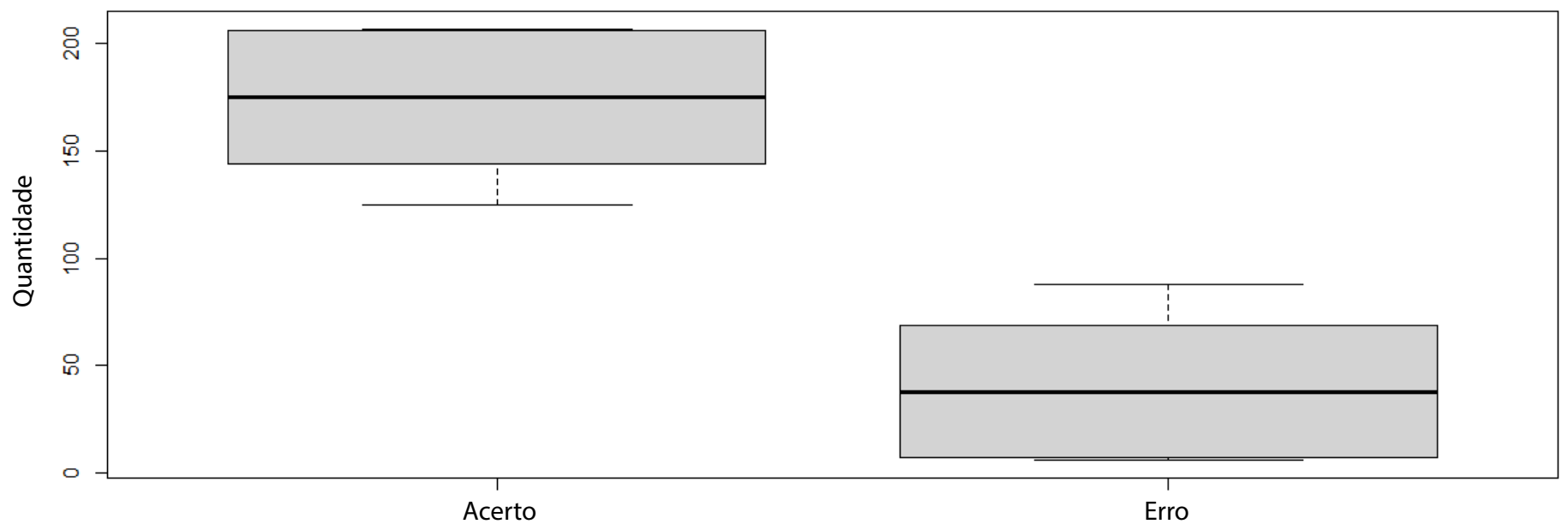




\section{DISCUSSÃO}

Sobre a discussão dos resultados, na questão 1 ("Qual é o seu nível de conhecimento sobre os transtornos da personalidade?") constatou-se que os participantes consideraram que o conhecimento geral deles sobre TP depois da aula foi superior a antes, o que indica que a estratégia da aula contribuiu com o saber autorrelatado dos alunos.

Na questão 2 ("Em termos quantitativos, como o uso de obras artísticas pode contribuir para o aprendizado de transtornos da personalidade?"), há uma discreta diferença entre os resultados de antes e depois, com aumento na pontuação média (1,35 ponto) e mediana (1 ponto) após a intervenção. Independentemente dessa pequena diferença, aparentemente, em média, os participantes antes da aula consideram que o cinema pode contribuir para o ensino dos TP e, após a aula, mantêm essa opinião.

Com relação à questão 3 ("Associe o transtorno da personalidade à sua definição"), há melhora significativa no desempenho dos estudantes ao conceituarem os TP antes e depois da aula, e há diferença nas médias de acertos e erros no momento depois ( $p$-valor $=3,718 \times 10-8$ ), o que indica desenvolvimento de conhecimento sobre os TP.

Considerando que no Brasil os cursos de graduação presenciais são constituídos por adultos jovens, com idades em torno de 21 anos no momento da matrícula e de 23 anos na conclusão do curso, propomos uma reflexão sobre o aprendizado para a população em questão ${ }^{12}$.

Uma pesquisa comparativa realizada com estudantes do ensino superior, brasileiros e norte-americanos, traz dados bastante relevantes no que tange aos estilos, às formas e aos métodos de aprendizagem. Ambos os grupos preferem o pensamento lógico, seguido de abordagens práticas e experiências. O pensamento imaginativo também é concebido como fundamental para a aprendizagem e inovação, embora em menor porcentagem que os anteriores. Os métodos intrapessoais e cinestésicos ocuparam posição de destaque entre os de maior preferência, e, entre os estudantes brasileiros, o linguístico também é ressaltado. $O$ consenso entre brasileiros e norte-americanos é que ambos almejam ocupar papel ativo em sala de aula e não apenas consumir informações passivamente ${ }^{13}$.

Diversos estudos realizados nas últimas décadas trouxeram inovações sobre a aprendizagem colocando em reflexão as concepções até então existentes sobre esse processo e a sua transposição nas práticas pedagógicas ${ }^{14}$.

O jovem adulto atual, para o qual as práticas pedagógicas do estudo em questão se direcionam, possui características singulares que o diferenciam das gerações anteriores. São descritos como "nativos digitais", nasceram em meio às modernas sociedades industrializadas e globalizadas. Estão mais independentes, conectados às grandes mídias e tecnologias, e mais bem informados do que as gerações anteriores. Produzem conteúdo e influenciam opiniões, transformando modelos de consumo ${ }^{15}$.

Os jovens estudantes estão, portanto, inseridos no contexto do atual cenário global, marcado pelo dinamismo e pela rapidez nas informações, ações e decisões. Por vezes, são ansiosos, imediatistas e multitarefas em sua maioria, e, para eles, as relações hierárquicas verticais estabelecidas fazem cada vez menos sentido ${ }^{16}$.

Assim, torna-se inviável ser professor e, consequentemente, elaborar planos e estratégias de ensino, desconsiderando as mudanças no contexto sociocultural no qual estamos inseridos e as influências que trazem para esses estudantes ${ }^{15}$.

Os principais cenários contemporâneos e seus personagens atuantes clamam por readequações das estratégias e metodologias de ensino das instituições do ensino superior, com o intuito de possibilitar que as metodologias de ensino-aprendizagem tradicionais sejam, progressiva e gradualmente, retiradas de cena. No entanto, a pedagogia da transmissão de conteúdo, de alunos depositários, ainda permanece hegemônica ${ }^{14}$.

As metodologias tradicionais de ensino caracterizam-se pela transferência de conhecimentos do professor, detentor do saber, para um estudante que assume o papel de depositário de informações, reconhecendo a realidade sem criticá-la. Considerando a formação de profissionais de saúde críticos e reflexivos que atuem em consonância com os princípios preconizados pelo Sistema Único de Saúde do Brasil, tais metodologias aparentam ter potencialidade limitada ${ }^{17}$.

O cinema se insere como estratégia de metodologia ativa que possibilita o aprendizado autorrelatado e observável dos estudantes de graduação. Em estudo anterior, o qual utilizou o cinema como recurso pedagógico para abordagem de conteúdos da psiquiatria, demonstrou-se a importância dessa arte na facilitação da visualização e compreensão do sujeito com transtornos mentais. $\mathrm{O}$ uso de cenas permitiu a projeção de si no outro, gerando um processo de identificação e consequente reflexão, um exercício empático que possibilitou a articulação dos conhecimentos teórico-práticos. A linguagem, imagem e narrativa cinematográfica permitiram que os estudantes enxergassem o que antes parecia pouco palpável e diminuíram a ansiedade relacionada ao momento da prática. Além disso, houve contribuição para minimizar o estigma relacionado ao indivíduo com transtorno mental, promovendo o olhar crítico sobre as intervenções realizadas ${ }^{18}$.

No que concerne aos transtornos de personalidade, o cinemeducation passa a ser uma opção ainda mais atrativa, pois, 
considerando a complexidade da temática, todas suas nuances e a multiplicidade de possibilidades diagnósticas, torna-se dificultosa a compreensão desses conceitos no ensino superior, em cargas horárias geralmente reduzidas. Assim, a integração sensorial dos estímulos recebidos por meio de filmes pode também favorecer o aprendizado sobre esses transtornos ${ }^{19}$.

\section{CONCLUSÕES}

Este estudo alcançou o objetivo proposto ao utilizar amostra estatisticamente significativa de estudantes e ao avaliar o conhecimento pré e pós-aula sobre TP, aplicando como intervenção uma aula com a estratégia cinemeducation. Os resultados mostraram que o método é efetivo para o ensino, havendo após a aula melhora autorreferida no conhecimento dos estudantes (questão 1) e melhora observável ao identificarem e conceituarem os TP (questão 3). Além disso, os estudantes referiram, em média, que a estratégia do uso de filmes contribuía para a aprendizagem, antes da aula, e mantiveram em média essa opinião, depois dela (questão 2).

Ademais, a discussão evidenciou que o uso dos filmes, com o intuito de subsidiar o aprendizado, respeita as características de estudantes da geração atual e é condizente com elas, uma vez que valoriza o uso de tecnologias em sala de aula e permite o aprendizado crítico-reflexivo, a participação ativa dos sujeitos e a experiência. Estimula a criatividade e torna possíveis os métodos de aprendizagem intrapessoal, cinestésico e linguístico. Ser inundado por intensos estímulos visuais e auditivos permite que esses jovens extrapolem as fronteiras das salas de aula e que o conhecimento adquirido rompa barreiras, e os conteúdos podem ser transpostos não só para o objetivo do ensino em graduação, como também para diversos aspectos de suas vidas.

As limitações do estudo se referem à escassez de filmes brasileiros contemporâneos com representações de todos os TP, não havendo cenas de personagens representativas para TP esquizotípica, dependente e esquiva neste estudo. Além disso, considera-se necessário estudo comparativo entre o método tradicional de ensino dos TP e o cinemeducation, de forma a investigar sua eficácia quando comparado às aulas tradicionais.

\section{CONTRIBUIÇÃO DOS AUTORES}

Os quatro autores trabalharam conjuntamente e se envolveram com todas as etapas da elaboração do presente artigo.

\section{FINANCIAMENTO}

Declaramos que não houve financiamento.

\section{REFERÊNCIAS}

1. Maia JMC, Castilho SM, Maia MC, Lotufo Neto F. Psicopatologia no cinema brasileiro: um estudo introdutório. Rev Psiquiatr Clín. 2005;32(6):319-23.

2. DiBartolo MC, Seldomridge LA. Cinemeducation: teaching end of life issues using feature films. J Geront Nurs. 2009;35(8):30-6.

3. Membrives MD, Isern MTI, Matheu MCL. Literature review: use of commercial films as a teaching resource for health sciences students. Nur Educ Tod 2016;36(1):264-7.

4. Borges TS, Alencar G. Metodologias ativas na promoção da formação crítica do estudante: o uso das metodologias ativas como recurso didático na formação crítica do estudante do ensino superior. Cairu em Revista. 2014;3(4):119-43.

5. Moreira JR, Ribeiro JBP. Prática pedagógica baseada em metodologia ativa: aprendizagem sob a perspectiva do letramento informacional para o ensino na educação profissional. Outras Palavras. 2016;12(2):93-114.

6. Hyler SE, Schanzer B. Using commercially available films to teach about borderline personality disorder. Bull Menninger Clin. 1997;61(4):458-68.

7. Gabbard GO, Gabbard K. Countertransference in the movies. Psycho Review. 1985;72(1):171-84.

8. Landeira-Fernandez J, Cheniaux E. Cinema e loucura. Porto Alegre: Artmed; 2010.

9. Louzã Neto MRL, Cordás T. Transtornos de personalidade. Porto Alegre: Artmed; 2009

10. American Psychiatry Association. Diagnostic and statistical manual of mental disorders - DSM-5. Washington: APA; 2013.

11. Honorato TG. Os transtornos da personalidade no cinema brasileiro [dissertação]. São Paulo: Universidade de São Paulo; 2018.

12. Instituto Nacional de Estudos e Pesquisas Educacionais Anísio Teixeira Censo da Educação Superior 2018: notas estatísticas [acesso em jan 2019]. Disponível em: http://inep.gov.br/web/guest/sinopses-estatisticas-daeducacao-superior.

13. Seemiller C, Grace M, Campagnolo PDB, Alves IMDR, Borba GSD. How generation Z college students prefer to learn: a comparison of U.S. and Brazil students. Journal of Educational Research \& Practice. 2019;9(1)349-68.

14. Lima VV. Espiral construtivista: uma metodologia ativa de ensinoaprendizagem. Interface. 2017; 21(61):421-34.

15. Silva RF. Do texto ao contexto: história, literatura e educação. Barbacena: EdUEMG; 2015.

16. Obregon SL, Facco ALR, Rodrigues GO, Marconatto DAB, Lopes LFD Geração Z: compreendendo as aspirações de carreira de estudantes de escolas públicas e privadas. Revista de Administração. 2016;15(26):84-108.

17. Lopes MSV, de Lima Filho FJR, Silva CL, Luciano EB, Cruz TG, Mota WS. O cinema como estratégia de metodologia ativa no ensino em enfermagem: relato de experiência. RSC online. 2017; 6(3):106-16.

18. Nicolau ARS, Camillo SO, Maiorino FT, Nóbrega MPSS. O cinema como recurso pedagógico na disciplina de enfermagem psiquiátrica. Rev Enferm Cent-Oeste Min. 2014;4(1):983-92.

19. Fonseca V. Desenvolvimento psicomotor e aprendizagem. Porto Alegre: Artmed; 2008.

\section{CONFLITO DE INTERESSES}

Declaramos não haver conflito de interesses. 